\title{
Decreased Efferents of Mesencephalic Raphe Nuclei to Striatum in Animal Model of Streptozocin-Induced Diabetes
}

\author{
Gholamreza Hassanzadeh ${ }^{1, *}$, Rostam Ghorbani ${ }^{2}$, Tahmineh Mokhtari ${ }^{1}$, Mohamad Bayat ${ }^{3}$, \\ Adib Zendedel ${ }^{1}$, Yosef Mohammadi ${ }^{1}$, Mitra Barzroodi \\ ${ }_{2}^{1}$ Department of Anatomy, Tehran University of Medical Sciences, Tehran, IR Iran \\ ${ }_{3}^{2}$ Department of Anatomy, Kermanshah University of Medical Sciences, Kermanshah, IR Iran \\ ${ }^{3}$ Department of Anatomy, Arak University of Medical Sciences, Arak, IR Iran \\ ${ }^{*}$ Corresponding author: Gholamreza Hassanzadeh, Department of Anatomy, Tehran University of Medical Sciences, Tehran, IR Iran. Tel.: +98-2188953008, Fax: +98-2166419072, E-mail: \\ hassanzadeh@tums.ac.ir.
}

Received: March 6, 2013; Revised: June 3, 2013; Accepted: September 14, 2013

\begin{abstract}
Background: Diabetes mellitus is a metabolic disease which affects the brain as well as other organs. Connection of mesencephalic raphe nuclei to striatum is important, and reduction of these projections can play a negative role in regulatory connection between them.

Objectives: The main objective of this study was to determine the effects of diabetes on projections of mesencephalic raphe nuclei to striatum of rats.

Materials and Methods: Forty eight male adult Wistar rats were divided into four groups (1.control, 2. two-month diabetic rats, 3. fourmonth diabetic rats and 4. six-month diabetic rats). HRP was injected into the dorsal and ventral striatum separately and after perfusion with normal saline, fixation, and postfix solutions, the brains were sectioned coronaly $(40 \mu \mathrm{m})$. Frozen sections were reacted with TMB and counterstained with $1 \%$ neutral red.

Results: This study showed reduction of labeled neurons in both mesencephalic raphe nuclei following induction of diabetes. After injection of HRP to ventral striatum this reduction was prominent in DR nucleus in comparison to median raphe projections to ventral striatum affected by diabetes more than that of those to dorsal striatum.

Conclusions: Diabetes mellitus decreases the projections of mesencephalic raphe nuclei to striatum.
\end{abstract}

Keywords: Diabetes Mellitus; Mesencephalon; Raphe Nuclei; Corpus Striatum; Streptozocin

\section{Background}

Diabetes mellitus is one of the increasing socio-medical problems (1). This metabolic disease involves the brain as well as beta cells of pancreas, muscles, and liver (2). There are several reports indicating that diabetes mellitus has a devastating effect on central nervous system, including a decrease in electrical connection between brain structures, impaired memory and learning, decrease in axonal transport, and difficulties in problem solving (3-8). Our previous study showed that the projections of raphe nuclei to hippocampal CA3 region in diabetic rats were less than nondiabetic group (9). Peripheral neuropathy, development and progression of depressive illness, and neural death are late complications of diabetes $(10,11)$. Mesencephalic raphe nuclei (dorsal and median) are the main serotonin resources innervating the forebrain (1214). The median raphe nucleus (MnR) exists rostral to the raphe pontis nucleus and consists of rostral and caudal subdivisions (9). Dorsal raphe (DR) nucleus is the largest serotonergic nucleus located in the ventral periaq- ueductal grey mater (PAG) which is divided into rostral and caudal parts $(9,15)$. Neostriatum or caudoputamen is somatotopically organized and involved in planning and modulation movement pathways $(13,16)$. This area is innervated by projections of raphe nuclei (17). Hyperglycemia results in increased blood flow in striatum and thalamus and causes hemichorea due to hyperactivity of neurons (18). Furthermore it impairs pro-oxidant/antioxidant balance and increases production of reactive oxygen species and lipid peroxidation in CNS (19). Therefore diabetes may affect survival and connections of neurons, and study different parts of the brain following induction of diabetes can be helpful in understanding the brain function.

\section{Objectives}

Because a decrease in the projections can cause a reduction in the neuron connections and neural messaging defects, this study was designed. The main objective of this study was to determine the effects of diabetes on projec-

Implication for health policy/practice/research/medical education:

This project evaluates the efferents of mesencephalic raphe nuclei to striatum in animal mode of streptozocin-induced Diabetes. As our knowledge, it is the first project in our country about this manner.

Copyright (C) 2014, Tehran University of Medical Sciences. This is an open-access article distributed under the terms of the Creative Commons Attribution License, which permits unrestricted use, distribution, and reproduction in any medium, provided the original work is properly cited. 
tions of mesencephalic raphe nuclei to striatum of rats.

\section{Materials and Methods}

\subsection{Animals}

48 male adult Wistar rats of 210 - 230 g body weight were divided into four groups (12 in each group) in simple randomized manner. They were housed under controlled temperature $\left(24^{\circ} \mathrm{C}\right)$ and lighting conditions (12 hours light/dark cycle). Food and water were available ad libitum. All animal care and procedures were in accordance with the ethics of ethical committee of Tehran University of Medical Sciences.

\subsection{Induction of Diabetes}

Diabetes was induced by single intraperitoneal injection of $60 \mathrm{mg} / \mathrm{kg}$ Streptozocin (STZ; Sigma. St Louis Mo, The USA; dissolved in $0.9 \%$ sterile Saline). Rats were divided into the following groups:1) control, 2) two month diabetic, 3) four month diabetic, and 4) six month diabetic. Hyperglycemia was confirmed using a strip-operated blood glucose sensor in a blood sample obtained by tail pick six days after the STZ injection.

\subsection{Track Tracing}

The animals were anesthetized by intraperitoneal injection of Ketamine (50 mg/kg) and Xylazine $(10 \mathrm{mg} / \mathrm{kg})$ and were given single stereotoxic injection of $0.5 \mu \mathrm{L} \mathrm{HRP}$ (Sigma, type VI) into the dorsal and ventral striatum separately as described in Paxinos Atlas. After 48 to 72 hours, which is the survival time the animals were killed with Ketamine and Xylazine, and perfused intracardiacly with $100 \mathrm{~mL}$ normal saline, $500 \mathrm{~mL}$ fixative solution (Glutaraldehyde $1.25 \%$ and Paraformaldehyde $1 \%$ in $0.2 \mathrm{M}$ phosphate buffer at $\mathrm{pH}=7.4$ ), and sucrose buffer $10 \%$, respectively. The brains were removed and post-fixed overnight $\left(4^{\circ} \mathrm{C}\right) .40 \mu \mathrm{m}$ frozen coronal sections were taken and reacted with tetra methyl benzidine (Sigma. St Louis. Mo, The USA) to reveal HRP activity. Sections were mounted on gelatinized slides and counterstained with $1 \%$ neutral red.

\subsection{Labeled Neurons Counting}

Serial sections were provided from tissue in $40 \mu \mathrm{m}$ slices and in every 10 , one slice was chosen for neuron counting. The extent of injection sites and distribution of retrogradely labeled neurons in dorsal and median raphe nuclei were counted by optika software.

\subsection{Statistical Analyses}

All data were expressed as mean values and their standard errors (S.E.). SPSS 16 software was chosen for analysis of the data. For analyzing the four groups of study one way-ANOVA test and Post Hoc of Tukey were chosen. The statistical significance level was set at $\mathrm{P} \leq 0.05$.

\section{Results}

\subsection{Blood Sugar and Body Weight}

The control animals showed a normal increase in body weight from $210 \pm 20$ to $410 \pm 5$ g; whereas, body weight decreased in the STZ-diabetic group from $210 \pm 20$ to $130 \pm$ $20 \mathrm{~g}$. In control group there was not any change in blood glucose level ( $80-110 \mathrm{mg} / \mathrm{dL}$ ), but in diabetic group blood glucose levels increased to $>300 \mathrm{mg} / \mathrm{dL}$.

\subsection{Projections of Dorsal Raphe and Median Raphe Nucleus Nuclei to Dorsal Striatum}

Injection of HRP into the dorsal striatum indicated a reduction in labeled neurons of the DR and MnR nuclei in all diabetic groups (Figure1 and 2). Reduced number of labeled neurons was significant in DR nucleus ( $P \leq 0.0001)$; whereas, no significant reduction was observed in MnR nucleus two months after the induction of diabetes. Decrease of labeled neurons in MnR became significant in the four and six month groups after injection of STZ respectively with $\mathrm{P} \leq 0.001$ and $\mathrm{P}$ $\leq 0.0001$ (Figure 3).

Figure 1. Projections of Dorsal Raphe Nucleus to Dorsal Striatum in Male Rats

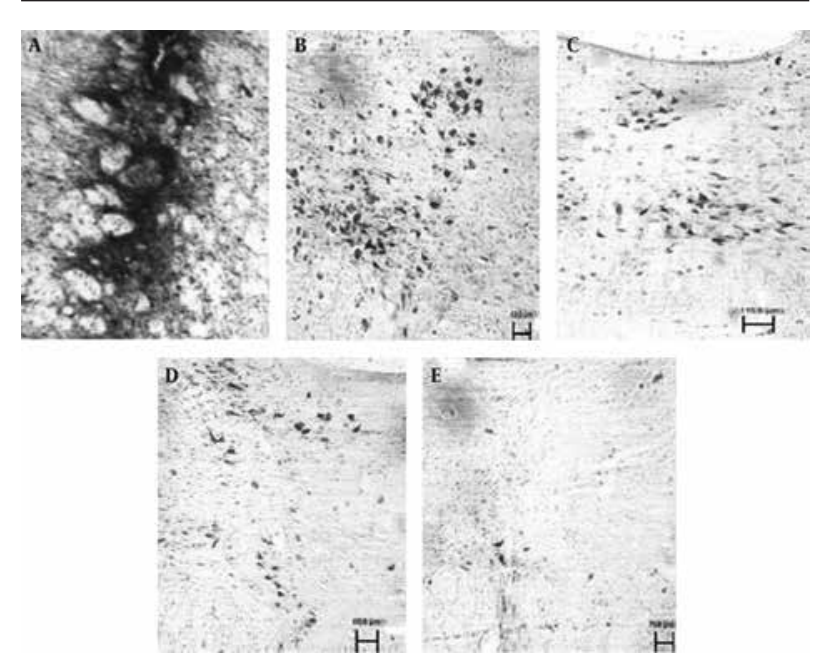

A) Photomicrograph of dorsal striatum after Injection of HRP, B) Labeled neurons in dorsal raphe nucleus of Control group, scale bar $83.3 \mu \mathrm{m}, \mathrm{C}$ ) Labeled neurons in dorsal raphe nucleus in two month diabetic group, scale bar $115.6 \mu \mathrm{m}$, D) Labeled neurons in dorsal raphe nucleus in four month diabetic group, scale bar $80.8 \mu \mathrm{m}$ and E) Labeled neurons in dorsal raphe nucleus in six month diabetic group, scale bar $75.8 \mu \mathrm{m}$ (X 100). 
Hassanzadeh G et al.

Figure 2. Projections of Median Raphe Nucleus to Dorsal Striatum in Male Rats
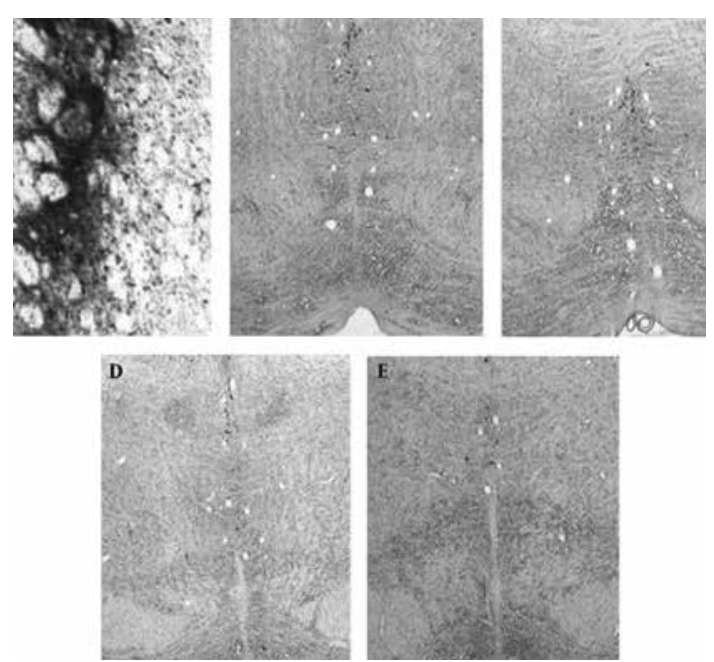

A) Photomicrograph of dorsal striatum after Injection of HRP, B) Labeled neurons in median raphe nucleus of Control group, C) Labeled neurons in median raphe nucleus in two month diabetic group, D) Labeled neurons in median raphe nucleus in four month diabetic group, and E) Labeled neurons in median raphe nucleus in six month diabetic group, scale bar $75.8 \mu \mathrm{m}(\mathrm{X} 100)$

Figure 3. Projections of Dorsal and Median Raphe Nuclei to Dorsal Striatum in Male Rats
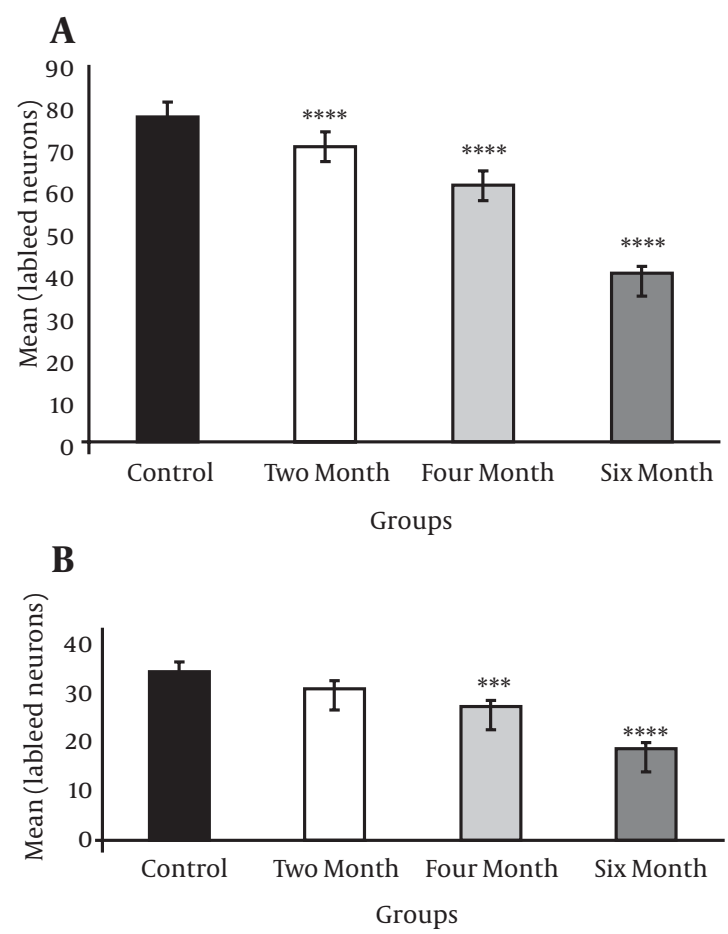

The number of labeled neurons in dorsal raphe nucleus (A) and median raphe nucleus (B) after injection of HRP into the dorsal striatum; values are mean $\pm \mathrm{SE}, \mathrm{N}=6,{ }^{* * *} \mathrm{P} \leq 0.001$ vs. Control-operated animals, ${ }^{* * *} \mathrm{P} \leq$ 0.0001 vs. Control-operated animals
Figure 4. Projections of Dorsal Raphe Nucleus to Ventral Striatum in Male Rats
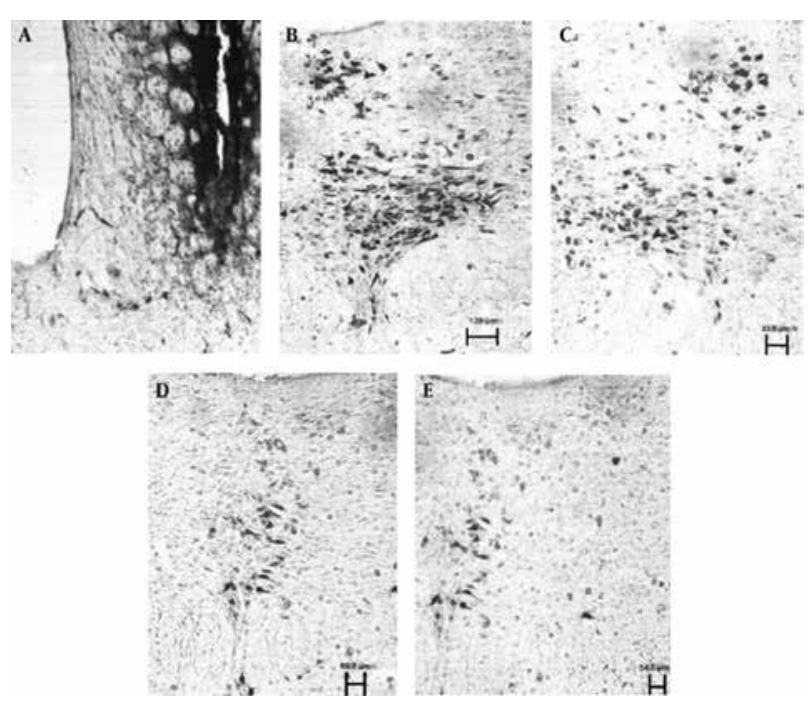

A) photomicrograph of ventral striatum after Injection of HRP, B) Labeled neurons in dorsal raphe nucleus of Control group, scale bar $128 \mu \mathrm{m}, \mathrm{C}$ ) Labeled neurons in dorsal raphe nucleus in two month diabetic group, scale bar $83.5 \mu \mathrm{m}$, D) Labeled neurons in dorsal raphe nucleus in four month diabetic group, scale bar $59.7 \mu \mathrm{m}$, and E) Labeled neurons in dorsal raphe

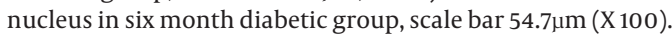

Figure 5. Projections of Median Raphe Nucleus to Ventral Striatum in Male Rats
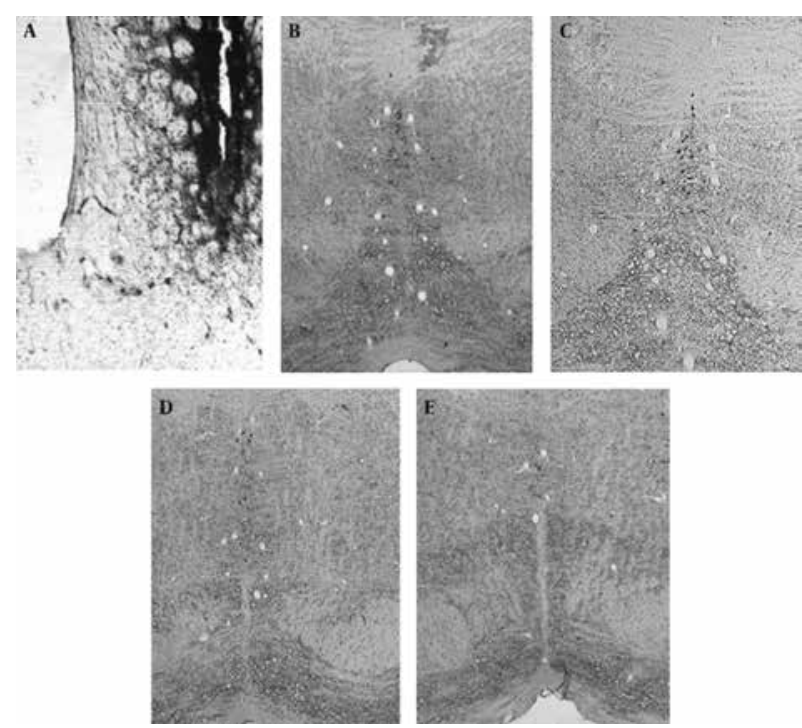

A) photomicrograph of ventral striatum after Injection of HRP, B) Labeled neurons in median raphe nucleus of Control group, C) Labeled neurons in median raphe nucleus in two month diabetic group, D) Labeled neurons in median raphe nucleus in four month diabetic group, and E) Labeled neurons in median raphe nucleus in six month diabetic group, scale bar $54.7 \mu \mathrm{m}$ (X100). 


\subsection{Projections of Dorsal and Median Raphe Nuclei to Ventral Striatum}

After injection of HRP into the ventral striatum, labeled neurons of DR and MnR nuclei decreased significantly, but this reduction was more significant in DR (P $\leq 0.0001)$ in comparison to $\mathrm{MnR}(\mathrm{P} \leq 0.01)$ two months after injection of STZ (Figure 4 and 5). Two months later, the number of decreased neurons was significant in both nuclei $(\mathrm{P} \leq 0.0001)$ (Figure 6).

Figure 6. The Number of Labeled Neurons in Dorsal Raphe Nucleus (A) and Median Raphe Nucleus (B) After Injection of HRP into the Ventral Striatum

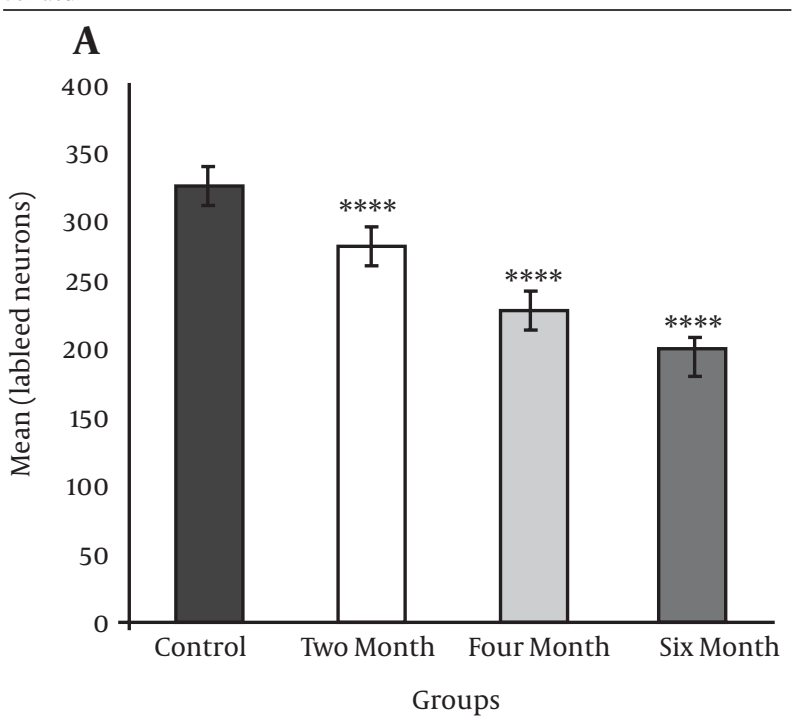

\section{B}

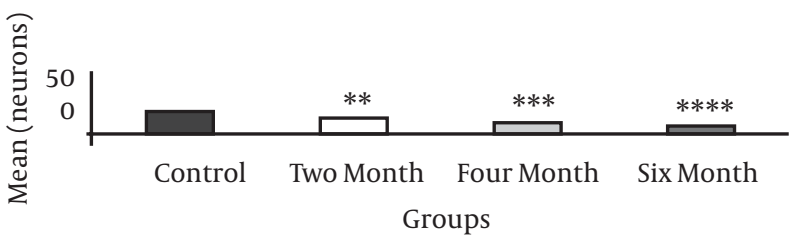

Values are mean $\pm \mathrm{SE}, \mathrm{N}=6,{ }^{* *} \mathrm{P} \leq 0.01$ vs. Control-operated animals, ${ }^{* * *} \mathrm{P}$ $\leq 0.001$ vs. Control-operated animals, ${ }^{* * * *} \mathrm{P} \leq 0.0001$ vs. Control-operated animals

\section{Discussion}

According to the results of this study, projections of DR nucleus to ventral striatum were more than of those to dorsal striatum in the control group. These findings are in accordance with previous studies (13-20). A significant decrease in DR efferents to dorsal and ventral striatum was found two months after the STZ injection. Our findings showed less labeled neurons in MnR nucleus in comparison to DR nucleus after injection of HRP into the dorsal and ventral striatum. This result is confirmed by our previous study (13). Two months after the STZ injection, MnR efferents to dorsal striatum were reduced, but it was not significant. However, this reduction became significant two months later. MnR projections to ventral striatum decreased significantly two months after diabetes induction, and this reduction remained significant after four months. The MnR projections to ventral striatum are affected by diabetes mellitus more than that of those to dorsal striatum. Study of Shapakov and colleagues showed that the level of serotonin in striatum is not significantly different between diabetic and nondiabetic rats (21). On the other hand, some studies indicate that induction of diabetes mellitus causes a decrease in serotonin level of the striatum $(22,23)$. We observed a decrease in projections but it is difficult to distinguish serotonergic neurons from non-serotonergics in our study. Tung and colleagues claimed that hyperglycemia can cause transient and reversible effects to basal nuclei functions (24). Furthermore, previous studies showed axonal transport reduction and cell death following diabetes mellitus (3). Our study indicated a decrease in projections of DR and MnR nuclei to striatum, which would not be transient or reversible if caused by cell death. Hence, more studies are required to clarify the exact relationship between diabetes mellitus and cell death in mesencephalic raphe nuclei $(25,26)$. Serotonergic neurons of DR mediate feeding behaviors. Ventral striatum can be activated by food stimuli because of its relation to limbic system $(25,26)$, and DR efferents to ventral striatum are more than of dorsal striatum. Therefore, diabetes-induced decrease in the number of efferents from DR to ventral striatum seems rational. The reduced number of projections from DR to dorsal striatum might influence the motor function (16), which needs to be confirmed by behavioral tests.

The numerous projections of DR and MnR nuclei to diverse regions of the brain suggested that these mesencephalic raphe nuclei are common targets of impairment in diabetes mellitus. We concluded that diabetes mellitus decreases the projections of mesencephalic raphe nuclei to the dorsal and ventral striatum.

\section{Acknowledgements}

The authors would like to thank the staff of Anatomy Department laboratories of Tehran University of Medical Sciences.

\section{Authors' Contribution}

Study concept and design: Gholamreza Hassanzadeh; Analysis and interpretation of data: Yosef Mohammadi, Mohamad Bayat and Adib Zendedel; Drafting of the manuscript: Gholamreza Hassanzadeh and Mitra Barzroodi Pour; Critical revision of the manuscript for important intellectual content: Rostam Ghorbani; Statistical analysis: Tahmineh Mokhtari. 


\section{Financial Disclosure}

There is no financial disclosure.

\section{Funding/Support}

This research in the Anatomy Department laboratories was supported by Tehran University of Medical Sciences, Tehran, IR Iran.

\section{References}

1. Hosseini A, Sharifzadeh M, Rezayat SM, Hassanzadeh G, Hassani $\mathrm{S}$, Baeeri M, et al. Benefit of magnesium-25 carrying porphyrinfullerene nanoparticles in experimental diabetic neuropathy. Int J Nanomedicine. 2010;5:517-23.

2. Gerozissis K. Brain insulin and feeding: a bi-directional communication. Eur J Pharmacol. 2004;490(1-3):59-70.

3. Abbate SL, Atkinson MB, Breuer AC. Amount and speed of fast axonal transport in diabetes. Diabetes. 1991;40(1):111-7.

4. Ceretta LB, Reus GZ, Rezin GT, Scaini G, Streck EL, Quevedo J. Brain energy metabolism parameters in an animal model of diabetes. Metab Brain Dis. 2010;25(4):391-6.

5. Cooray GK, Hyllienmark L, Brismar T. Decreased cortical connectivity and information flow in type 1 diabetes. Clin Neurophysiol. 2011;122(10):1943-50.

6. Jolivalt CG, Calcutt NA, Masliah E. Similar pattern of peripheral neuropathy in mouse models of type 1 diabetes and Alzheimer's disease. Neuroscience. 2012;202:405-12.

7. van Duinkerken E, Klein M, Schoonenboom NS, Hoogma RP, Moll AC, Snoek FJ, et al. Functional brain connectivity and neurocognitive functioning in patients with long-standing type 1 diabetes with and without microvascular complications: a magnetoencephalography study. Diabetes. 2009;58(10):2335-43.

8. Zsombok A, Smith BN. Plasticity of central autonomic neural circuits in diabetes. Biochim Biophys Acta. 2009;1792(5):423-31.

9. Hassanzadeh G, Zendedel A, Akbari M, Jemeie SB, Mehrannia K. Effect of diabetes on median and dorsal raphe nuclei efferent fibers to CA3 hippocampal area in rat. Acta Med Iran. 2010;48(1):1-8.

10. Ali S, Stone MA, Peters JL, Davies MJ, Khunti K. The prevalence of co-morbid depression in adults with Type 2 diabetes: a systematic review and meta-analysis. Diabet Med. 2006;23(11):1165-73.

11. Hosseini A, Abdollahi M, Hassanzadeh G, Rezayat M, Hassani S, Pourkhalili N, et al. Protective effect of magnesium-25 carrying porphyrin-fullerene nanoparticles on degeneration of dorsal root ganglion neurons and motor function in experimental diabetic neuropathy. Basic Clin Pharmacol Toxicol. 2011;109(5):381-6.

12. Baker KG, Halliday GM, Hornung JP, Geffen LB, Cotton RG, Tork I. Distribution, morphology and number of monoamine-synthe- sizing and substance P-containing neurons in the human dorsal raphe nucleus. Neuroscience. 1991;42(3):757-75.

13. Behzadi GR, Hassanzadeh G. Projections of dorsal and median raphe nuclei to dorsal and ventral striatum. Acta Med Iran. 2007;45(5):339-44.

14. Morin LP, Meyer-Bernstein EL. The ascending serotonergic system in the hamster: comparison with projections of the dorsal and median raphe nuclei. Neuroscience.1999;91(1):81-105.

15. Waselus M, Valentino RJ, Van Bockstaele EJ. Collateralized dorsal raphe nucleus projections: a mechanism for the integration of diverse functions during stress.J Chem Neuroanat. 2011;41(4):266-80.

16. Gireesh G, Kumar TP, Mathew J, Paulose C. Enhanced muscarinic M1 receptor gene expression in the corpus striatum of streptozotocin-induced diabetic rats. J Biomed Sci. 2009;16:38.

17. Fibiger HC, Miller JJ. An anatomical and electrophysiological investigation of the serotonergic projection from the dorsal raphe nucleus to the substantia nigra in the rat. Neuroscience. 1977;2(6):975-87.

18. Nabatame H, Nakamura K, Matsuda M, Fujimoto N, Shio H. Hemichorea in hyperglycemia associated with increased blood flow in the contralateral striatum and thalamus. Intern Med. 1994;33(8):472-5.

19. Reagan LP. Diabetes as a chronic metabolic stressor: causes, consequences and clinical complications. Exp Neurol. 2012;233(1):68-78.

20. Vertes RP. A PHA-L analysis of ascending projections of the dorsal raphe nucleus in the rat. J Comp Neurol.1991;313(4):643-68.

21. Shpakov AO, Kuznetsova LA, Plesneva SA, Bondareva VM, Guryanov IA, Vlasov GP, et al. Decrease in functional activity of Gproteins hormone-sensitive adenylate cyclase signaling system, during experimental type II diabetes mellitus. Bull Exp Biol Med. 2006;142(6):685-9.

22. Broderick PA, Jacoby JH. Diabetes-related changes in L-tryptophan-induced release of striatal biogenic amines. Diabetes. 1988;37(7):956-60.

23. Broderick PA, Jacoby JH. Central monoamine dysfunction in diabetes: psychotherapeutic implications: electroanalysis by voltammetry. Acta Physiol Pharmacol Latinoam. 1989;39(3):211-25.

24. Tung CS, Guo YC, Lai CL, Liou LM. Irreversible striatal neuroimaging abnormalities secondary to prolonged, uncontrolled diabetes mellitus in the setting of progressive focal neurological symptoms. Neurol Sci. 2010;31(1):57-60.

25. Batterham RL, ffytche DH, Rosenthal JM, Zelaya FO, Barker GJ, Withers DJ, et al. PYY modulation of cortical and hypothalamic brain areas predicts feeding behaviour in humans. Nature. 2007;450(7166):106-9.

26. Chechlacz M, Rotshtein P, Klamer S, Porubska K, Higgs S, Booth D, et al. Diabetes dietary management alters responses to food pictures in brain regions associated with motivation and emotion: a functional magnetic resonance imaging study. Diabetologia. 2009;52(3):524-33. 